\title{
Vaccinations against influenza and pneumococcus in children with diabetes: telephone questionnaire survey
}

\author{
Patrick Davies, Chinedu Nwokoro, Mira Leigh
}

Since 1992, the Department of Health has encouraged people in certain at risk groups to have vaccinations against influenza and pneumococcus. ${ }^{1}$ The vaccinations are recommended for patients with diabetes, heart disease, or chronic respiratory disease (including asthma), and all people older than 65 . The campaign has no lower age limit; children are included by default. We investigated uptake of pneumococcus and influenza vaccine in all children with diabetes in our area. We also audited advice given about vaccination by consultants and paediatric diabetic nurse specialists in the hospitals in the region of the Eastern Deanery of the United Kingdom.

\section{Participants, methods, and results}

We interviewed all children attending Bedford Hospital's diabetes clinic by telephone with 12 structured questions. We sent a questionnaire with a stamped self addressed envelope to children whom we could not contact by telephone. We telephoned all lead consultants and paediatric diabetic nurse specialists who specialise in diabetes in the region and asked whether they advise vaccinating children who have diabetes.

We found 75 children who are currently being followed up. We successfully interviewed 49 by telephone. We contacted the remaining 26 by letter; 11 replied (table). The overall response rate was 60/75. A total of 25 were boys, median age was 13 years, and diagnosis of diabetes had happened at median age 5 years. All the healthcare professionals responded.

A total of 56/60 children had had their diphtheria, tetanus, and pertussis vaccine, 58/60 their measles, mumps, and rubella (MMR) vaccine, and 55/57 their preschool booster (three children were too young). Professional advice given to patients varied within and between hospitals. In $9 / 17$ hospitals, the consultant and the nurse gave different advice.

\section{Comment}

Mass vaccination should only be advised if the benefits outweigh the risks. (About a third of children would be classified as at risk and be affected.) Healthcare advisers need to be careful when advising a population measure, as any further erosion of confidence in public health advice may lead to an irretrievably poor relationship with the public. Some non-peer reviewed reports of adverse reactions to the influenza vaccination in adults are appearing on the internet. ${ }^{23}$ Therefore, any such advice needs to be based on good evidence.

Although vaccinating children who have serious chronic conditions where any deterioration in lung function could be life threatening-for example, cystic fibrosis-makes sense, routine immunisation for people in the categories defined by the Department of Health has implications for resources and ethics. Paediatricians need to reach consensus.
Vaccinations had by children with diabetes and advised by their healthcare professionals, Bedford Hospital

\begin{tabular}{lccc} 
& & \multicolumn{2}{c}{ Vaccinated against } \\
\cline { 3 - 4 } Question & Total & Influenza & Pneumococcus \\
\hline Children & & & NA \\
\hline Vaccinated in $2001(95 \% \mathrm{Cl})$ & $56^{*}$ & $14(10$ to 18$)$ & NA \\
\hline Vaccinated in $2002(95 \% \mathrm{Cl})$ & 60 & $28(23$ to 33$)$ & 1 \\
\hline Ever vaccinated & 60 & $\mathrm{NA}$ & 2 \\
\hline Healthcare professionals & & & 4 \\
\hline Consultants advising vaccination & 17 & 11 & \\
\hline Nurses advising vaccination & 17 & 10 & \\
\hline
\end{tabular}

\section{NA=Not applicable}

${ }^{*}$ Four children had a diagnosis of diabetes for less than one year.

We reviewed medical journals and found no studies of children with diabetes showing increased morbidity or mortality associated with infection by influenza or pneumoccocus. We got some references suggesting a theoretical benefit of vaccination for adults with diabetes but no reports of vaccine effectiveness from the Department of Health. ${ }^{45}$

Our subjects were not against vaccinations as such, as the data for routine childhood immunisations show. The median age was 13 , however, so they predate the controversy surrounding the measles, mumps, and rubella vaccine.

In $9 / 17$ hospitals the advice given by the consultant differed to that given by the nurse. This inconsistency reflects either lack of awareness or poor confidence among specialists in guidelines conceived without an evidence base. Also, communication within specialist teams is obviously lacking. Such inconsistency both within and between hospitals can only lead to confused patients and loss of trust in the medical profession.

UK healthcare providers need to reach an evidence based consensus regarding vaccination against influenza and pneumococcus in children at risk. Despite national guidelines, responsibility for patients remains with individual practitioners. Each team needs a clear policy to avoid confusing diabetic patients.

We thank Julia Brown for her help with identifying patients. Contributors: PD had the concept, did the patient arm of the study, and drafted the paper. CN did the professional arm of the study. ML oversaw the study and is guarantor.

Funding: None.

Competing interests: None declared.

Ethical approval: Not needed.

\footnotetext{
1 Department of Health. Immunisation against infectious disease. London: Stationery Office, 1992.

Flu vaccine reaction media stories. www.whale.to/vaccine/flu6.html Flu vaccine reaction
(accessed 30 Oct 2003).

Free case evaluation: influenza (flu) vaccine. New York: Parker and Free case evaluation: influenza (flu) vaccine. New York: Parker and
Waichman. www.yourlawyer.com/practice/overview.htm?topic=Influenza $\% 20$ (Flu) $\% 20$ Vaccine (accessed 30 Oct 2003).

4 Moss SE, Klein R, Klein BEK. Cause specific mortality in populationbased study of diabetes. Am J Public Health 1991;81:1158-62.

5 Bouter KP, Diepersloot RJ, van Romunde LK, Uitslager R, Masurel N, Hoekstra JB, et al. Effect of epidemic influenza on ketoacidosis, pneumonia and death in diabetes mellitus: a hospital register survey of 1976-79 in the Netherlands. Diabetes Res Clin Pract 1991;12;61-8.

(Accepted 3 September 2003)
}

Department of Paediatrics, Bedford Hospital NHS Trust, Bedford MK42 9DJ

Patrick Davies paediatric registrar Chinedu Nwokoro senior house officer

Mira Leigh consultant paediatrician Correspondence to: P Davies daviespatrick@ hotmail.com

BMJ 2004;328:203 